\title{
113 THE DIAGNOSTIC UTILITY OF RT-QUIC IN SPORADIC CJD
}

Louise Davidson, Alison Green, Lynne McGuire, Robert G Will, Richard Knight. Edinburgh

10.1136/jnnp-2014-309236.113

Sporadic CJD (sCJD) remains the commonest type of CJD. Currently, there is not a disease specific antemortem test available for the diagnosis of $\mathrm{sCJD}$ with postmortem remaining the definitive method of diagnosis. Current diagnostic criteria rely on clinical presentation in association with the MRI, EEG and CSF 14-3-3 protein. However, none of these tests are specific for CJD. Atypical presentations can be diagnostically challenging and there can be a delay in diagnosis which can be distressing for relatives. This identified a need for a disease specific, reliable diagnostic test that can provide an earlier and more accurate diagnosis.

A recently developed assay called real time quaking induced conversion (RT-QuIC) exploits the seeded conversion of normal prion protein to the abnormal form and therefore detects disease associated prion protein in the CSF. Based on recent evidence, it has been suggested that RT-QuIC has the potential to identify cases that conventional techniques such as CSF 14-3-3 may miss and potentially contribute to an earlier and more accurate diagnosis. We will present the results of a prospective analysis of the utility of RT-QuIC in routine clinical practice and whether it has any added benefit over current pre-mortem diagnostic methods. 